\title{
Actividades institucionales para el uso del tiempo libre de los alumnos
}

\author{
Institutional activities for the use of students' free time
}

\author{
Yasmín María Camargo Casadiego \\ yasminca292010gmail.com \\ Código ORCID: 0000-0003-4314-6694 \\ Universidad del Zulia. Venezuela
}

\author{
Maigualida Bejas Monzant \\ maigualidabejas@yahoo.com \\ Código ORCID: 0000-0003-3030-9298 \\ Universidad del Zulia, Venezuela
}

\section{RESUMEN}

La investigación tuvo como propósito analizar las actividades institucionales para el uso del tiempo libre de los alumnos. Metodológicamente se abordó un enfoque cuantitativo de tipo descriptiva, el diseño no experimental, de campo. La población estuvo conformada por los directivos y docentes de planta de la I.E.D. José Laborde Gnecco. Este estudio determinó la presencia de notables carencias y deficiencias en la planificación institucional, con un desempeño muy por debajo de unos límites deseables, en el desarrollo de actividades para el uso apropiado del tiempo libre de los estudiantes. Se concluyó que la institución no posee una planificación adecuada de actividades para el uso del tiempo libre de los alumnos y que pueda ser beneficioso para su desarrollo integral bajo la orientación y asesoría idónea de los docentes del plantel.

Palabras clave: Planificación; actividades institucionales; tiempo libre

\section{ABSTRACT}

The purpose of the research was to analyze institutional activities for the use of students' free time. Methodologically, a descriptive quantitative approach was approached, the non-experimental design, in the field. The population was made up of the directors and plant teachers of the I.E.D. José Laborde Gnecco. This study determined the presence of notable deficiencies and deficiencies in institutional planning, with a performance far below desirable limits, in the development of activities for the appropriate use of students' free time. It was concluded that the institution does not have adequate planning of activities for the use of the students' free time and that it may be beneficial for their integral development under the guidance and suitable advice of the teachers of the campus

Keywords: planning, institutional activities, free time 


\section{INTRODUCCIÓN}

Actualmente, los jóvenes, en esta sociedad moderna, se enfrentan a diversos problemas sociales, que han deteriorado su calidad de vida y han afectado su salud como son: el sedentarismo ocasionado por la adicción al internet y a los dispositivos tecnológicos, la drogadicción, las relaciones sexuales de manera precoz e irresponsable, la agresividad, la violencia, etc. Según las Bases del Plan Nacional de Desarrollo 2010- 2014. Titulado "Prosperidad para Todos" (2011)

En los preadolescentes (12-14 años) y aquellos propiamente adolescentes (14-18 años), la problemática se centra principalmente en la vulneración $\mathrm{o}$ inadecuada realización de sus derechos sexuales y reproductivos (embarazo de adolescentes) y a la vinculación a actividades como bandas y pandillas, organizaciones criminales y reclutamiento por parte de grupos armados irregulares. (p. 263)

Lo que puede llevar a pensar que gran parte de esta problemática es ocasionada por el mal uso que los jóvenes hacen de su tiempo libre, el cual vivencian en lugares poco apropiados para ellos como son las calles, los parques, las casas de videos juegos, los centros comerciales, etc. Alejados de cualquier acción educativa $\mathrm{y}$, por el contrario, llenos de situaciones de riesgo que comprometen su integridad. Como lo afirma la ONU (2008):

... riesgos y vulnerabilidades que se ocultan tras muchas formas de maltrato y abuso: el abuso y la explotación sexual; la trata; los trabajos peligrosos; la violencia; la vida o el trabajo en la calle; el efecto de los conflictos armados, en particular el empleo de niños por fuerzas y los grupos armados; las prácticas nocivas como la mutilación y ablación de los genitales femeninos $y$ el matrimonio precoz; la falta de acceso a la justicia; y el internamiento innecesario, entre otras. Un entorno de protección acelera el progreso del desarrollo infantil $y$ mejora la salud, la educación y el bienestar de los niños, así como su capacidad de adaptación para convertirse en padres, ciudadanos y miembros de provecho de la sociedad. (p.2)

En nuestra sociedad, las instituciones ofrecen al estudiante muy poca posibilidad de ocupar el tiempo libre de manera provechosa para su desarrollo integral. Sobre todo, ahora que se está implementando la jornada extensiva en la mayoría de las escuelas como lo plasman García Fernando (1996) y Guardia (2000) en (Nuviala, Ruíz, y García, 2003, pp. 9-26): 
El tiempo libre de los adolescentes va aumentando al mismo ritmo que el resto de la sociedad. Existe una tendencia a agrupar la jornada escolar o a crear una jornada intensiva, con el consiguiente aumento del tiempo libre. En este tiempo es donde la sociedad debe intervenir para facilitar la ocupación del niño, en actividades que favorezcan su desarrollo integral.

La Institución Educativo Distrital José Laborde Gnecco, al igual que muchas instituciones del distrito de Santa Marta, se han interesado muy poco en planificar y desarrollar diversas actividades fuera de su rutina académica que conlleven a la buena utilización del tiempo libre por parte de los jóvenes y han venido funcionando como lugares ajenos de las realidades y necesidades en que conviven los alumnos. Como se encuentra plasmado en el Plan Nacional de Desarrollo 2010- 2014. "Prosperidad para Todos":

... es importante contar con estrategias, programas $\mathrm{y}$ proyectos encaminados a ofrecer espacios para el aprovechamiento del tiempo libre, en donde además se refuercen aspectos del desarrollo personal, el liderazgo y la participación ciudadana juvenil y la integración con la comunidad, el control social. (2011, p. 2)
Ante esta realidad, el problema objeto de estudio de la investigación se centró en la falta o poca planificación de programas o proyectos en la Institución Distrital José Laborde Gnecco que incluyan la recreación, la cultura y el deporte, como complemento de los aprendizajes de manera amena, como lo dice la Ley General de Educación (Decreto 1860 de 1994 artículo 57 inciso 4):

El Proyecto Educativo
Institucional de cada institución
educativa debe fijar un tiempo
dedicado a actividades lúdicas,
culturales, deportivas y sociales, de
contenido educativo orientadas
por pautas curriculares, según el
interés del estudiante, tiempo que
no podrá ser inferior a diez (10)
horas semanales.

Una de las principales causas de este objeto de estudio es que no se está concientizando ni incentivando a los jóvenes para que hagan uso de este tiempo, de una manera formativa, creativa y lúdica favoreciendo la creación de espacios de socialización, de integración y de formación, como lo dice la Ley 375 de 1997 en su artículo 9: "El Estado garantiza el ejercicio del derecho de los jóvenes a la recreación, práctica de deporte y aprovechamiento creativo del tiempo libre. Para esto dispondrá de los recursos físicos, económicos y humanos necesarios". 
Es por esto que las instituciones educativas deben motivar a los padres, a las diferentes asociaciones y a otros organismos para que colaboren entre sí y de esta manera ofrecer variadas opciones a los estudiantes donde puedan practicar diferentes actividades deportivas, recreativas, artísticas y culturales haciendo uso de su infraestructura.

Para Douglas Marr citado por Trigo, A. (1990), las escuelas tienden a concentrar y proveer pequeños aspectos recreativos y actividades más que a capacitar a los alumnos con actividades y habilidades sociales que ellos mismos organicen, seleccionen y participen en elegir, con vista a los años posteriores a la escuela.

Es importante resaltar que las actividades planeadas por cada institución deben responder a las expectativas, necesidades y requerimientos que tiene la comunidad y los alumnos y debe estar acorde con las posibilidades locativas.

Cabe anotar también que las actividades deben estar articuladas al Proyecto Educativo Institucional, contribuyendo de esta forma, a un proceso integral de formación de los jóvenes. Es decir, las actividades proyectadas en la institución deben estar sustentadas en un quehacer educativo de acuerdo al modelo pedagógico de ésta, para que de verdad estas prácticas tengan un carácter realmente educativo $\mathrm{y}$ formativo.
Por esta razón el PEI debe ser diseñado no solo para desarrollarse en los espacios y tiempos de la escuela, sino que debe traspasar su acción a periodos diferentes a los escolares. Las puertas de las instituciones deben estar abiertas y sus horarios deben extenderse para trabajar actividades importantes que contribuyan en la formación integral de los estudiantes. De esta manera la escuela se proyectará más allá de sus instalaciones logrando así un cambio o transformación en su contexto, ya que las actividades formativas que se trabajarán serán totalmente diferentes, aún más si están conducidas a desarrollar nuevos hábitos saludables de vida.

Es por esto que el uso del tiempo libre se ha convertido en un problema para su análisis: Según Weber (1969), Racionero (1983), Muñoz M. (1983), Pedro (1984), se han planteado interrogantes como ¿Qué hacer con el tiempo libre y para qué?, ¿Qué sentido darle a la gran cantidad de tiempo libre del cual disponen los adolescentes?, ¿Cómo hacer que el tiempo libre se convierta en un elemento esencial en la vida de los jóvenes?

En tal sentido se requiere una investigación profunda donde se hagan análisis y reflexiones pertinentes desde el campo educativo y formativo sobre el tiempo libre de los jóvenes y la falta de actividades planificadas en las instituciones para el buen uso de éste, como un factor que debe estar 
involucrado en los proyectos educativos para el desarrollo integral del estudiante.

Objetivo General: Analizar las actividades institucionales para el uso del tiempo libre de los alumnos de la I.E.D. José Laborde Gnecco.

\section{Objetivos específicos:}

- Diagnosticar las actividades culturales implementadas en la institución para el uso del tiempo libre de los alumnos de la I.E.D. José Laborde Gnecco.

- Determinar las actividades institucionales deportivas que se aplican para el uso del tiempo libre de los alumnos de la I.E.D. José Laborde Gnecco.

- Describir las actividades recreativas que se desarrollan para el uso del tiempo libre de los alumnos en la I.E.D. José Laborde Gnecco.

- Identificar las actividades artísticas existentes en la institución para el uso del tiempo libre de los alumnos de la I.E.D. José Laborde Gnecco.

\section{Actividades Institucionales para el uso del tiempo libre}

A continuación se establecen las bases conceptuales sobre actividad desde el punto de vista psicológico y pedagógico. Desde el punto de vista psicológico, Merani (1979) afirma que la actividad: "se refiere al conjunto de fenómenos de la vida activa, como los instintos, las tendencias, la voluntad, el hábito, etc., que constituye una de las tres partes de la psicología clásica, junto con la sensibilidad y la inteligencia" (p. 4)

Otro autor que maneja la actividad desde el enfoque psicológico es Leóntiev (2005), quien convierte a la actividad como objeto de la psicología y la define como un determinado proceso real que consta de un conjunto de acciones y operaciones que permiten al hombre relacionarse con el mundo para adaptarse a él y para poder transformarlo, así mismo determinó que el objetivo y el motivo son los componentes principales de la actividad.

Asimismo, Talízina (2002) manifiesta que dentro de los rasgos de la actividad coincide el motivo y el objetivo, asumiendo que el motivo es el detonante de la actividad que se determina por el objetivo. Para poder llegar al cumplimiento de tal objetivo se requiere de las acciones como componentes primordiales de la actividad.

De acuerdo a los planteamientos anteriores, toda actividad va acompañada de la búsqueda de un logro, el cual debe estar seguido de acciones esenciales para su consecución y las acciones son procesos orientados por el motivo de la actividad.

Según el enfoque psicológico, entonces, una acción que realiza un sujeto, en este caso el alumno, está dirigida a un objeto material o ideal, con 
el fin de dar cumplimiento a un objetivo previamente determinado por el profesor.

Por otro lado, Talízina (1984) expresa que en la actividad planificada desde el punto de vista pedagógico, el motivo y el objetivo deben coincidir, es decir, la actividad debe satisfacer una necesidad cognoscitiva y por consiguiente sus motivos han de ser también cognitivos. De lo anterior se deduce que el aprendizaje para que sea una actividad, o una acción, siempre debe de estar concebida desde un objetivo y un motivo, aspectos esenciales en la estructura a la que se hace referencia.

Las actividades institucionales para el uso del tiempo libre son aquellas que se planifican dentro del currículo educativo de carácter obligatorio con metas y objetivos claros y con opciones variadas para el estudiante (culturales, deportivas, recreativas, etc.), donde él pueda hacer uso de su tiempo libre de manera provechosa logrando con ellas un desarrollo integral, explotando su máxima potencialidad. Camargo (2016). Sustentado en: Munné (1960), Weber (1963), Dumazedier (1967), Monsiváis,
(1976): Fantova, (1990), García Montes (2001).

\section{MÉTODO}

El estudio se enmarcó en un tipo de investigación descriptiva. Según esta tipología se describe, determinan, establecen y caracterizan propiedades y rasgos de la realidad estudiada. En este caso: analizar cómo están los lineamientos institucionales a través de docentes y directivos de la I.E.D. José Laborde Gnecco.

El diseño de Investigación se basó en un diseño no experimental y de campo. Los instrumentos aplicado fueron los cuestionarios a los involucrados (docentes y directivos) en su ambiente real (I.E.D. José Laborde Gnecco) sin ninguna alteración ni preferencias y sin ofrecer estímulos para obtener determinadas respuestas con el fin de obtener resultados verídicos y correctos.

La población estudio fue constituida por los docentes y directivos de la I. E.D. José Laborde Gnecco, las características comunes que poseen los profesores y directivos de las instituciones tales como las que se menciona a continuacion: 
Tabla 1. Características comunes que poseen los profesores y directivos de las instituciones

\begin{tabular}{lccc}
\hline Planteles & $\begin{array}{c}\mathbf{N}^{\circ} \\
\text { de docentes }\end{array}$ & $\begin{array}{c}\mathbf{N}^{\circ} \\
\text { de directivos }\end{array}$ & $\begin{array}{c}\mathbf{N}^{\circ} \text { total de } \\
\text { docentes } \\
\text { directivos }\end{array}$ \\
\hline I.E.D JOSE LABORDE GNECCO & 46 & 3 & 49 \\
I.E.D SIMÓN BOLÍVAR “GAIRA” & 35 & 3 & 38 \\
I.E.D CAMILO TORRES & 64 & 4 & 68 \\
I.E.D GABRIELA MISTRAL & 20 & 2 & 22 \\
I.E.D BEATRIZ VIVES DE CAMPO & 16 & 2 & 18 \\
TOTAL & 181 & 14 & 195
\end{tabular}

Fuente: S.E.D. (2016) Secretaria de Educación de Santa Marta, planta personal docente 2016, oficina de Recursos Humanos.

Tabla 2. Población de estudio

\begin{tabular}{cll}
\hline Jornadas & Docentes & Personal directivo \\
\hline Mañana y tarde & 46 & 3 \\
& & \\
\hline
\end{tabular}

Las características de la muestra fueron:

1.- Docentes de planta que laboran en la I.E.D. José Laborde Gnecco en ambas jornadas y 2.- Coordinadores de planta que laboran en la I.E.D. José Laborde Gnecco en ambas jornadas.

Se tomó como muestra intencional o también definida como intencional $\mathrm{u}$ opinático, que según Arias (2006, p. 85), "en este caso los elementos son escogidos con base en criterios o juicios preestablecidos por el investigador".
En el caso de una población homogénea, la representatividad de tal muestra puede considerarse satisfactoria". Para este caso, los principales criterios que privaron que representen a la misma y que respondan a los objetivos de la investigación, la muestra de este estudio reúnen todos las mismas características, ser docentes y directivos de la I.E.D. José Laborde Gnecco. 
Tabla 3. La muestra

\begin{tabular}{lcc}
\hline Niveles & Docentes & Personal directivo \\
\hline Nivel pre-escolar & 1 & \\
Nivel primaria & 12 & 1 \\
Nivel secundaria & 18 & 1 \\
Nivel media & 15 & 1 \\
Total & 46 & 3 \\
\hline
\end{tabular}

La Técnica e Instrumento de Recolección de Datos fue la encuesta, ya que con esta se obtiene información directa de la muestra a través de los cuestionarios de preguntas cerradas basadas en la escala de Likert, el instrumento de recolección de datos que se aplicó fue un cuestionario autoadministrado. Para ello se elaboró un cuestionario conformado por 45 Ítemes, con una escala tipo Likert (5: Siempre; 4: Casi Siempre; 3: Algunas Veces; 2: Casi Nunca; 1: Nunca), que permitió la obtención de información sobre todas y cada una de las dimensiones de la variable en estudio. El instrumento fue diseñado en base a las dimensiones e indicadores de medición de la variable en estudio.

Para la validez de los instrumentos se procedió a la revisión de los mismos por parte de cuatro expertos conocedores de la temática de estudio; quienes, de acuerdo a su experiencia, una vez revisados los instrumentos, conjuntamente con un formato de validación, establecieron la pertinencia de cada ítem con las variables, dimensiones e indicadores, de modo que al ser revisado por los validadores se derivó a las correcciones sugeridas por los mismos, para luego generar el cuestionario definitivo.

La confiabilidad de los cuestionarios de la investigación se obtuvo mediante la aplicación del Método Alfa de Cronbach, que consiste en la expresión matemática mediante la fórmula aportada por Chávez (2004), de la siguiente manera: $r_{k k=\frac{K}{K-1}} 1-\varepsilon \frac{S^{2} i}{S^{2} t}$

Dónde: $\mathrm{r} \mathrm{kk}=$ coeficiente $\mathrm{de}$ confiabilidad,$K=$ Número de ítems del instrumento, $S^{2} i=$ varianza de los puntajes de cada ítem, $S^{2} t=$ varianza de los puntajes totales y

$$
1=\text { Constante }
$$

Para el cuestionario autoadministrado, la confiabilidad se determinó obteniendo el coeficiente Alfa de Cronbanch, aplicando el programa estadístico SPSS versión 19 a la base de datos, obtenida de los resultados del cuestionario aplicado.Para establecer la aceptación o no del coeficiente de confiabilidad, se utilizó el siguiente baremo de interpretación: 
Tabla 4. Interpretación del coeficiente de confiabilidad.

\begin{tabular}{ll}
\hline RANGO & \multicolumn{1}{c}{ MAGNITUD } \\
\hline 0,81 a 1,00 & Muy alta \\
0,61 a 0,80 & Alta \\
0,41 a 0,60 & Moderada \\
0,21 a 0,40 & Baja \\
0,01 a 0,20 & Muy baja \\
\hline
\end{tabular}

Fuente: Ruiz (2007)

El valor del coeficiente Alfa de Cronbach obtenido fue de $\alpha=0,72$. Este resultado le confiere, según el cuadro anterior, una confiabilidad alta al instrumento utilizado.

La técnicas de análisis de la información de la investigación fueron los datos cuantitativos por tanto se procedió a realizar con ellos procedimientos estadísticos necesarios para interpretar la información dada como la tabulación de la información obtenida del instrumento, mediante la utilización de estadística descriptiva. Los datos recolectados con el cuestionario autoadministrado, atendiendo a la variable de estudio, fueron tratados y resumidos a través del uso de porcentajes (frecuencias porcentuales), manejados a partir de las convenciones admitidas universalmente. $\mathrm{Al}$ introducir los porcentajes en el análisis de los datos, se trata de proporciones que se multiplican por 100 .

Para este propósito se utilizó el Programa SPSS, versión 19 estándar para Windows. Los insumos que se determinaron en el estudio fueron las Tablas de Distribución de Frecuencias y los Diagramas de Barras; todo esto se realizó de cada uno de los elementos que conformo la variable, establecido previamente.

Por otra parte, para el estudio de cada uno de los indicadores se tomó el promedio de los ítems que conformaron cada indicador, según lo estipulado, las dimensiones con los indicadores que la conformaron y el de la variable en cuenta al promedio de las dimensiones que la conforman. Dado que los resultados estuvieron entre un valor mínimo, que en este caso es el correspondiente a Nunca (1), y un valor máximo, que es el correspondiente a Siempre (5), al tener en cuenta que en algunos casos los resultados no fueron números naturales, nace la necesidad de diseñar un baremo que tipifique todos los posibles resultados a la escala original, para su posterior análisis. Dicho baremo se presenta a continuación: 
Tabla 5. Baremo de transformación escalar de los indicadores, dimensiones y variable

\begin{tabular}{llc}
\hline RANGO & ALTERNATIVA & ESCALA \\
\hline $5,00-4,20$ & SIEMPRE & 5 \\
$4,19-3,39$ & CASI SIEMPRE & 4 \\
$3,38-2,58$ & ALGUNAS VECES & 3 \\
$2,57-1,77$ & CASI NUNCA & 2 \\
$1,76-1,00$ & NUNCA & 1 \\
\hline
\end{tabular}

Fuente: Camargo (2017)

\section{RESULTADOS}

Los hallazgos se reflejaron por dimensiones a fin de hacer una mejor demostración de los efectos. La Dimensión Actividades Culturales evidenció que los valores que revelan una situación alarmante para esta dimensión, puesto que se obtuvo una ausencia $(0 \%)$ de respuestas favorables.
Esto muestra serias deficiencias institucionales en la planificación y ejecución para el desarrollo de actividades artísticas y culturales por parte de los estudiantes, bajo la conducción de sus docentes. Esto reviste mayor preocupación, al tratarse de una institución educativa enclavada en un Distrito Turístico, Cultural e Histórico como lo es Santa Marta.

Tabla 6. Dimensión actividades culturales

\begin{tabular}{cccc}
\hline Categorías de Respuesta & Frecuencia & Porcentaje & Porcentaje acumulado \\
\hline 2,00 & 1 & 2,0 & 2,0 \\
3,00 & 48 & 98,0 & 100,0 \\
Total & 49 & 100,0 & \\
\hline
\end{tabular}

Fuente: SPSS aplicado a la base de datos (2017)

En este sentido, si se quiere lograr la formación integral en los alumnos, se debe permitir a los mismos adquirir conocimientos y desarrollar habilidades en todas las dimensiones del ser humano incluyendo la cultural, con el objetivo de tener un mejor perfil académico $\mathrm{y}$ personal. Por esto, las actividades culturales deben planificarse en la institución para el tiempo libre como objetivos primordiales para la participación social, la interrelación entre los miembros de la comunidad y el trabajo solidario y voluntario por un bien común. 
Dimensión Actividades Deportivas: Al observar los valores de la Tabla 7, se obtiene que sólo el $24,5 \%$ de los profesores encuestados proporcionan respuestas consideradas favorables (categorías Siempre y Casi Siempre). Al comparar este porcentaje con el valor mínimo establecido del $80 \%$, se nota que es muy inferior a éste, evidenciando que no hay una tendencia favorable del comportamiento general del grupo de estudio con respecto a esta dimensión. Esto evidencia la presencia de marcadas deficiencias institucionales para la planificación y puesta en ejecución de actividades deportivas que realicen los estudiantes en su tiempo libre.

Tabla 7. Dimensión actividades deportivas

\begin{tabular}{cccc}
\hline Categorías de Respuesta & Frecuencia & Porcentaje & Porcentaje acumulado \\
\hline 3,00 & 37 & 75,5 & 75,5 \\
4,00 & 12 & 24,5 & 100,0 \\
Total & 49 & 100,0 & \\
\hline
\end{tabular}

Fuente: SPSS aplicado a la base de datos (2017)

Lo anterior difiere del pensamiento que la práctica deportiva debe ser considerada como un derecho humano. En La Carta Olímpica, se expresa que toda persona debe tener la posibilidad de practicar deporte sin discriminación de ningún tipo y dentro del espíritu olímpico, que exige comprensión mutua, solidaridad y espíritu de amistad y fair play.

Es importante la planificación de actividades deportivas, ya que se refieren a la relevancia de la actividad física en la vida del ser humano, mejorando su calidad de vida a través del desarrollo de sus relaciones sociales, convirtiéndose para los jóvenes en actividades de suma importancia que deben desarrollarse e incentivarse en las instituciones.

La Dimensión Actividades Recreativas se obtuvo un $63,3 \%$ de respuestas consideradas favorables. Este valor es menor que el $80 \%$ mínimo exigido para considerar la existencia de una tendencia favorable en este renglón. Sin embargo, al compararla con el desempeño en las restantes dimensiones, se observa un notorio incremento en el porcentaje de respuestas favorables. Pero sigue manteniéndose la tendencia deficitaria institucional en cuanto a la planificación y desarrollo de actividades recreativas bajo la conducción de los docentes del grupo de estudio. 
Tabla 8. Dimensión actividades recreativas

\begin{tabular}{cccc}
\hline Categorías de Respuesta & Frecuencia & Porcentaje & Porcentaje acumulado \\
\hline 3,00 & 18 & 36,7 & 36,7 \\
4,00 & 31 & 63,3 & 100,0 \\
Total & 49 & 100,0 & \\
\hline
\end{tabular}

Fuente: SPSS aplicado a la base de datos (2017)

No coincide lo anterior con el pensamiento de Molina (1991) que afirma que la recreación es una dimensión de la vida a la que cada vez más se le reconoce su importancia, sobre todo en un mundo donde también necesitamos cada vez más espacios para la vivencia de significados asociados con nuestra realización física, mental, emocional y espiritual, la recreación es uno de esos espacios.

Las actividades recreativas promueven estilos de vida saludables proporcionando a los adolescentes experiencias positivas que se desearan repetir, lo que lleva a un aumento de la actividad física y mejorar la salud mental $\mathrm{y}$ física. Los adolescentes desarrollan habilidades interpersonales útiles y se relacionan con amigos a través de actividades recreativas compartidas mientras se desarrollan la autoestima y la confianza. La recreación promueve el desarrollo de actitudes sociales positivas y facilita las amistades de toda la vida con otras personas que comparten intereses similares. Mientras practican actividades recreativas, los adolescentes están fuera de las calles y alejados de influencias negativas.

Es necesario entonces y de suma importancia desarrollar las actividades recreativas en las instituciones ya que éstas constituyen el medio principal para el proceso educativo del tiempo libre y el desarrollo de los conocimientos, habilidades, motivaciones, actitudes, comportamientos y valores en relación con el empleo positivo de éste tiempo.

La dimensión Actividades Artísticas: La Tabla y correspondiente a esta dimensión arrojan como resultado un exiguo $4,1 \%$ de respuestas favorables. Este valor es muy inferior a $80 \%$, porcentaje mínimo establecido en el criterio de decisión para considerar que hay una tendencia favorable en este rubro. Este hallazgo revela la existencia de marcadas carencias institucionales en la planificación y realización de actividades artísticas guiadas por los docentes del grupo de estudio. 
Tabla 9. Dimensión actividades artísticas

\begin{tabular}{cccc}
\hline Categorías de Respuesta & Frecuencia & Porcentaje & Porcentaje acumulado \\
\hline 3,00 & 47 & 95,9 & 95,9 \\
4,00 & 2 & 4,1 & 100,0 \\
Total & 49 & 100,0 & \\
\hline
\end{tabular}

Fuente: SPSS aplicado a la base de datos (2017)

Lo anterior difiere de afirmaciones como la de Lowenfeld, (1980, p.20) que nos dice: "La educación artística como parte esencial del proceso educativo, puede ser muy bien la que responda por la diferencia que existe entre un ser humano creador y sensible y otro que no tenga capacidad para aplicar sus conocimientos, que no disponga de recursos espirituales y que encuentre dificultades en sus relaciones con el ambiente. En un sistema educacional bien equilibrado se acentúa la importancia del desarrollo integral de cada individuo, con el fin de que su capacidad creadora potencial pueda perfeccionarse.

Las actividades artísticas en las instituciones promueven en los jóvenes el desarrollo de la capacidad creativa, la autoestima, la disposición de aprender, la capacidad de trabajar en equipo o el pensamiento abstracto, todo esto con los objetivos de sensibilizar y disfrutar las manifestaciones artísticas sin necesidad de ser expertos.
Por ello, la planificación de actividades artísticas son fundamentales en el desarrollo integral de los alumnos y una opción prioritaria para tener en cuenta en el tiempo libre de los jóvenes para realizar en su jornada extendida o extraescolar.

Los resultados globales para la variable Actividades Institucionales para el Uso del Tiempo Libre no pueden ser más desalentadores: $0 \%$ de respuestas favorables (categorías Siempre y Casi Siempre). Este valor indica la presencia de notables carencias y deficiencias en las planificaciones institucionales, con un desempeño muy por debajo de unos límites deseables en el desarrollo de actividades para el uso apropiado del tiempo libre de los estudiantes, bajo la orientación y asesoría idónea del plantel de docentes de la institución objeto de estudio.

Tabla 10. Variable actividades institucionales para el uso del tiempo libre

\begin{tabular}{cccc}
\hline Categorías de Respuesta & Frecuencia & Porcentaje & Porcentaje acumulado \\
\hline 3,00 & 49 & 100,0 & 100,0
\end{tabular}

Fuente: SPSS aplicado a la base de datos (2017) 
Este resultado está en contraste con autores como Fantova (1990), quien afirma que el tiempo libre debe estar relacionado con el desarrollo integral de los jóvenes y el cual debe ser gratificante y nunca impuesto u obligado, el tiempo libre debe ser un tiempo liberador, generador de procesos de personalización y transformación social, tiempo condicionado y de potencialidad relativa, pero apostando por explotar todo lo que tenga.

Aquí Fantova se refiere al tiempo libre como un tiempo que ayuda al desarrollo personal e integral de los jóvenes ayudándolos a explotar todas sus capacidades, el cual debe ser un tiempo ajeno a las labores cotidianas, pero sin dejar de ser importante para el ser humano. Este tiempo de calidad es sobre el cual se realizará nuestra investigación y es el objeto de nuestro estudio.

Cabe anotar que este tiempo libre al desarrollarse en una institución debe contener siempre una finalidad. Monsiváis (1976), "como concepto contemporáneo el tiempo libre, como construcción capitalista, supone la existencia de un programa, de un sistema que tiende a uniformar actividades, metas y objetivos" ( $p$ 147).

Este concepto de Monsiváis se acerca al tema de estudio pues aborda el tiempo libre en el ámbito educativo y está enmarcado en el logro de unos objetivos y propósitos inmersos en un currículo.

Es decir que la planificación de actividades institucionales para el uso del tiempo libre es una necesidad social que está en relación con lo que denominamos calidad de vida, aspecto éste directamente relacionado con el tiempo libre. Se puede afirmar que la calidad de vida que experimenta una persona está ligada al tiempo libre.

\section{CONCLUSIONES}

Una vez analizados los resultados de la investigación se procede a exponer las afirmaciones que se extraen de los hallazgos en consideración a los objetivos de la investigación:

En relación al primer objetivo que tiene que ver con la dimensión de actividades culturales implementadas en la institución, los resultados arrojan un bajo porcentaje de respuestas que se ubican en la categoría de favorables ya que el valor obtenido está muy por debajo del porcentaje mínimo exigido.

Por tanto, se evidencian carencias significativas en la planificación y desarrollo de esta dimensión. Lo que revela una situación alarmante, pues esto muestra serias deficiencias institucionales en la planificación de actividades culturales, que son de capital importancia para el logro de la integración cultural de la comunidad con la institución educativa. 
Estas falencias en la aplicación de actividades institucionales culturales por parte de los docentes del grupo de estudio. Reviste gran preocupación, al tratarse de una institución educativa enclavada en un Distrito Turístico, Cultural e Histórico como lo es Santa Marta. Lo cual debe ser motivo de una profunda reflexión por parte de todos los actores vinculados al desarrollo de estas actividades, tanto docentes de aula como personal directivo.

En relación al segundo objetivo se observó que en algunos indicadores de la dimensión, como actividades al aire libre y mantenimiento a la salud, se infiere una ligera tendencia favorable que indica que no hay carencias notorias en la planificación de estos dos indicadores y que los docentes las aplican apropiadamente.

Sin embargo, se muestra, en el resultado general del grupo de estudio con respecto a esta dimensión, que no hay una tendencia favorable; lo que evidencia, la presencia de marcadas deficiencias institucionales para la planificación y puesta en ejecución de actividades deportivas que realicen los estudiantes en su tiempo libre.

Este resultado es preocupante, ya que estas actividades extracurriculares resultan atractivas para los estudiantes, puesto que se refieren a las prácticas deportivas y el ejercicio corporal. Se exhiben, por lo tanto, claras deficiencias en la planificación y ejecución de esta actividad por parte del grupo de estudio.

En relación al tercer objetivo con respecto a la dimensión recreativa, al compararla con el desempeño en las restantes dimensiones, se observó un notorio incremento en el porcentaje de respuestas favorables, pero solo en el indicador de entretenimiento.

Sin embargo, la dimensión recreativa, de manera general, muestra que el porcentaje de las respuestas favorables, al compararlo con el porcentaje establecido como mínimo, resulta ser inferior.

Esto indica la existencia de deficiencias en la planificación $y$ ejecución de actividades recreativas $y$ que sigue manteniéndose la tendencia deficitaria institucional en cuanto a la planificación y desarrollo de estas actividades bajo la conducción de los docentes del grupo de estudio.

En cuanto al cuarto objetivo relacionado con las actividades artísticas, el resultado del estudio revela la presencia de serias carencias en la planificación, conducción e implementación de actividades artísticas para los estudiantes por parte de los profesores encuestados, ya que, no logra llegar al porcentaje establecido como mínimo para que pudiera considerarse la existencia de una tendencia favorable. 
Esto indica que el grupo de estudio presenta marcadas carencias institucionales en la planificación y aplicación de estrategias que conduzcan al desarrollo de esta dimensión en el tiempo libre guiadas por los docentes del grupo de estudio.

Este hallazgo debe llamar a una profunda y seria reflexión, puesto que al tratarse de una institución educativa que está ubicada en una región con un alto potencial turístico, no debe descuidarse la planificación, uso y promoción de la dimensión de actividades artísticas en el tiempo libre de los estudiantes, que resalten estos elementos culturales y propicien la identidad local y regional de una ciudad con un gran atractivo turístico.

Con referencia al objetivo general de analizar las actividades institucionales para el uso del tiempo libre de los alumnos, se determinó la presencia de notables carencias y deficiencias en la planificación institucional, con un desempeño muy por debajo de unos límites deseables, en el desarrollo de actividades para el uso apropiado del tiempo libre de los estudiantes.

Lo que lleva a concluir que la institución no posee una planificación adecuada de actividades para el uso del tiempo libre de los alumnos, que desarrolle en ellos todos sus potenciales y que pueda ser beneficioso para su desarrollo integral bajo la orientación y asesoría idónea de los docentes del plantel.

\section{REFERENCIAS}

Arias, F. (2006). Proyecto de investigación: introducción a la metodología científica. Caracas: Episteme

Bases del Plan Nacional de Desarrollo (2010- 2014). Prosperidad para Todos, 2011, p. 263

Bases del Plan Nacional de desarrollo (2014-2018). "Todos por un nuevo país" 2016, p. 77

Dumazedier, J. (1967). Hacia una civilización del ocio. Barcelona: Estela

Fantova, F. (1990). "Evaluación de programas de intervención en el tiempo libre con personas con minusvalía en el Reino Unido, Italia y Francia"

García, Fernando. (1996) Guardia. (2000). Citado por Alberto Nuviala, Francisco Ruíz Juan y M. ${ }^{a}$ Elena García Montes. (2003). "Gestión deportiva, ocio activo y turismo"

García Montes, M. (2001) Actitudes y comportamientos de la mujer granadina ante la práctica fisica de tiempo libre (Tesis Doctoral). Universidad de Granada, España

Ley General de Educación República de Colombia (1994). Decreto 1860 de 1994 artículo 57 inciso 4

Ley 375 de 1997. Artículo 9. Tiempo Libre 
Lowenfeld, V. (1980).Desarrollo de la capacidad creadora. Buenos Aires: Kapelusz

Merani, A. (1979). Diccionario de psicología. México D. F: Grijalbo

Molina, M.C. (1991) Educación sexual en el cole. Madrid. Diseño

Monsiváis, C. (1976). Imágenes del tiempo libre en días de guardar". México: Era

Munné, F. (1960). Psicosociología del tiempo libre. México: Trillas

Organización para Naciones Unidas. (2008). "Estrategia de protección de la infancia del UNICEF". Consejo Económico y Social. Fondo de las Naciones Unidas para la Infancia. Junta Ejecutiva. Período de sesiones anual de 2008. Pág. 2.

Ruiz, J.I. (2007): El sector no lucrativo en España: una visión reciente. Fundación BBVA.
Talízina, N. (1988). Psicología de la Enseñanza. Moscú: Editorial Progreso.

Talízina, N. (2002). La Teoría de la Formación de las Acciones Mentales de P. Y. Galperin. Conferencia dictada en el seminario internacional de psicología, Actualidad, aplicaciones y perspectivas de la teoría histórico-cultural; Puebla, México

Trigo, A. (1990) Juventud, Tiempo Libre y Educación en Galicia. Tesis Doctoral, UNED, Madrid

Weber, E. (1963) Das frezeitproblem. Anthropologische - pädagogische Untersuchung. Manchen: Emts Reinhardt Verlang. (El problema del tiempo libre: Estudio antropológico y Pedagógico. Madrid: Editorial Nacional, 1969) 